\title{
Acolhimento noturno: expectativas dos profissionais de saúde mental
}

\section{User embracement: expectations of the mental health professionals}

\author{
Christiane Okazaki ${ }^{1}$, Márcia Aparecida Ferreira de Oliveira², \\ Heloísa Garcia Claro ${ }^{3}$, Heloisa Barboza Paglione ${ }^{4}$, Ricardo Soares ${ }^{5}$
}

OKAZAKI, C.; OLIVEIRA, M. A. F.; CLARO, H. G.; PAGLIONE, H. B.; SOARES, R. Acolhimento noturno: expectativas dos profissionais de saúde mental. Rev. Ter. Ocup. Univ. São Paulo, v. 21, n. 2, p. 166-173, maio/ago. 2010.

\begin{abstract}
RESUMO: Este estudo teve como objetivo identificar as expectativas dos profissionais de saúde mental de um Centro de Atenção Psicossocial, da cidade de São Paulo, quanto à implantação do serviço 24 horas (acolhimento noturno). Trata-se de uma pesquisa descritiva que se caracteriza pela interrogação direta dos indivíduos sobre os significados que um determinado assunto produz em suas vidas. Participaram deste estudo 11 atores, das seguintes categorias: enfermeira, médicos, psicólogos e assistentes sociais. Para a análise dos dados foram utilizados os passos da análise hermenêutica dialética: ordenação, classificação e análise final. Realizou-se a caracterização da população e a classificação das informações obtidas em expectativas positivas e negativas. A análise final considera que a implantação de um serviço de acolhimento noturno representa mais um recurso no arsenal terapêutico para o tratamento de pessoas portadoras de transtorno mental grave e persistente, que permite a atenção contínua.
\end{abstract}

DESCRITORES: Acolhimento; Psiquiatria; Saúde mental. Serviços de saúde/recursos humanos; Serviços de saúde mental/recursos humanos; Pessoal de saúde.

\footnotetext{
1. Enfermeira, membro do Grupo de Estudos em Álcool e outras Drogas da Escola de Enfermagem da USP. e-mail: chris.okazaki@gmail. com.

2. Enfermeira, Livre Docente do Departamento de Enfermagem Materno-Infantil e Psiquiátrica da Escola de Enfermagem da USP, líder do Grupo de Estudos em Álcool e Outras Drogas da Escola de Enfermagem da USP. e-mail: marciaap@usp.br.

${ }^{3}$. Enfermeira, Mestre e Doutoranda em Ciências pelo Programa de Pós Graduação em Enfermagem da Escola de Enfermagem da USP, membro do Grupo de Estudos em Álcool e outras Drogas da Escola de Enfermagem da USP. e-mail: heloisa.claro@usp.br.

${ }^{4}$. Estudante de graduação em Enfermagem da Escola de Enfermagem da USP. membro do Grupo de Estudos em Álcool e Outras Drogas da Escola de Enfermagem da USP. e-mail: heloisa.paglione@usp.br.

5. Psicólogo, Mestrando pelo Programa de Pós-Graduação em Enfermagem da Escola de Enfermagem da USP, membro do Grupo de Estudos em Álcool e Outras Drogas da Escola de Enfermagem da USP. e-mail: rhssoares@hotmail.com.

Endereço para correspondência: Av. Dr. Enéas de Carvalho Aguiar, 419 - 05403-000 - São Paulo - SP.
} 


\section{INTRODUÇÃO}

A Reforma Psiquiátrica vem acontecendo em alguns países desde o final da Segunda Guerra Mundial e tomou vigor no Brasil a partir da década de 80, do século passado. O movimento da Reforma Psiquiátrica inseriu o tema da desinstitucionalização na pauta da política pública e, consequentemente, o da mudança radical no modelo de atenção à saúde mental. Essa mudança liga-se às estratégias de difusão e ampliação das inovações institucionais surgidas e desenvolvidas no campo da saúde mental, como os Centros de Atenção Psicossocial - CAPS (ARANHA; SILVA, 1997).

Os Centros de Atenção Psicossocial são unidades de referência de saúde mental, com equipe multiprofissional e têm como missão tratar de forma intensiva os portadores de transtornos mentais graves, na comunidade, junto às suas famílias, evitando a internação psiquiátrica integral e promovendo a reabilitação psicossocial. Segundo Saraceno (1999), reabilitação psicossocial é definida "como sendo um processo que determina o aumento da capacidade do usuário de utilizar oportunidades e recursos, num percurso que faça crescer sua contratualidade psicológica e social, construindo o direito pleno da cidadania e a constituição material de tal direito".

No final da década de 80 , do século passado, ocorre uma relativa transformação da assistência psiquiátrica, sobretudo, com o desenvolvimento das primeiras experiências substitutivas à assistência hospitalar, como a criação, em 1987, do Centro de Atenção Psicossocial ${ }^{1}$ Professor Luis da Rocha Cerqueira (CAPS) ${ }^{2}$, pela Secretaria Estadual de Saúde de São Paulo, e dos Núcleos de Atenção Psicossocial (NAPS), em 1989, pela Prefeitura de Santos (PITTA, 1994).

Estas transformações ocorrem na medida em que o tratamento fornecido deve ser dimensionado segundo as necessidades do indivíduo e possa transformar as condições de vida do paciente. Para Pitta (1994), esses indícios de transformação podiam e podem ser observados por experiências inovadoras e com êxito em curso, em vários municípios brasileiros. Assim, demonstra-se a viabilidade da implantação de uma rede integrada de serviços, composta por diversas modalidades de recursos assistenciais e comunitários, que começam a prescindir dos recursos manicomiais. Nessas localidades, novas práticas se consolidam centradas na convivência com as diferenças e no respeito ao direito de cidadania das pessoas portadoras de sofrimento psíquico (MÂNGIA; BARROS, 2009).

A crescente utilização dos leitos psiquiátricos em hospitais gerais é outro fato que indica uma tendência promissora voltada à reversão do modelo assistencial asilar (a queda do número de leitos em hospitais psiquiátricos expressa a crise do modelo assistencial hospitalocêntrico); a mudança no financiamento dos serviços de saúde mental. A ampla divulgação, nos meios de comunicação de massa, da situação atual da assistência psiquiátrica e da denúncia de violação dos direitos humanos dos pacientes internados são aspectos cruciais para modificação da cultura de exclusão e do estigma frente à loucura e ao doente mental (BRASIL, 2007).

Durante a aprovação da Lei $\mathrm{n}^{\circ} 10.216$, de 6 de abril de $2001^{3}$, ocorreu o fechamento significativo de hospitais psiquiátricos que não atendiam minimamente a critérios básicos de assistência. Concomitantemente, estava sendo implantado um serviço substitutivo ao modelo tradicional; os chamados serviços de atenção diária, com unidades em todo o país e com leitos reservados em hospitais gerais (BRASIL, 2002).

A Portaria/SNAS n 224, de 29 de janeiro de 1992, refere-se aos Centros de Atenção Psicossocial (CAPS), unidades de saúde com uma estrutura de atendimento à saúde mental a qual vem substituindo o modelo hospitalocêntrico, e tem por finalidade oferecer tratamento a pessoas acometidas por doença mental (BRASIL, 2002). São, ainda, unidades assistenciais que podem funcionar 24 horas por dia, durante os sete dias da semana ou durante cinco dias úteis, das 8 às 18 horas, segundo definições do órgão gestor local.

Devido à tendência de reestruturação do atendimento às pessoas com transtornos psíquicos, os hospitais

\footnotetext{
1. "Centro de Atenção Psicossocial: nome emprestado aos Centros existentes na Nicarágua. A aplicação brasileira dessa designação foi sugerida pela Dra. Ana Maria Fernandes Pitta”, Diretora da Divisão de Ambulatórios da Coordenadoria de Saúde Mental do Estado de São Paulo (1983-1986). GOLDBERG, J. I. Reabilitação como processo - o Centro de Atenção Psicossocial - CAPS. In: PITTA, A. (Org.). Reabilitação psicossocial no Brasil. São Paulo: Hucitec, 1996. p. 33.

2 "Dr. Luis da Rocha Cerqueira (1911-1984): médico psiquiatra, nascido em Alagoas, formado na Bahia, com longa história institucional no hospício da Tamarineira, Recife. Semeador de reformas psiquiátricas em Recife, Salvador, Rio de Janeiro, Ribeirão Preto e São Paulo. Coordenador de Saúde Mental do Estado de São Paulo (1972)." Ibid., p. 33.

3 Lei n ${ }^{\circ} 10.216$, de 06 de abril de 2001, que dispõe sobre a proteção e os direitos das pessoas portadoras de transtornos mentais e redireciona o modelo assistencial em saúde mental (Ministério da Saúde, 2004).
} 
OKAZAKI, C. et al. Acolhimento noturno: expectativas. Rev. Ter. Ocup. Univ. São Paulo, v. 21, n. 2, p. 166-173, maio/ago. 2010.

psiquiátricos estão sendo desativados. Nessa perspectiva, foi necessário encontrar alternativas para os usuários que necessitassem de atendimento no período noturno (no qual os Centros de Atenção Psicossocial não funcionam) pudessem receber os cuidados e atenção que demandavam, sem a privação da autonomia, anulação de seu poder contratual e seu afastamento do núcleo familiar. Foram esses motivos que levaram a implantação dos Centros de Atenção Psicossocial 24 horas - CAPS III (BRASIL, 2002).

Segundo a Portaria GM/MS no 336, de 19 de fevereiro de $2002^{4}$, o serviço de atenção psicossocial com capacidade operacional para atendimento em municípios com população acima de 200.000 habitantes pode se constituir em serviço ambulatorial de atenção contínua, durante 24 horas, diariamente, incluindo feriados e finais de semana (BRASIL, 2002).

No último quadriênio, foram reduzidos cerca de 1700 leitos hospitalares ao ano no Brasil. O serviço CAPS III funciona como uma contrapartida ao fechamento destes leitos, uma vez que é necessário assegurar a continuidade da atenção, entretanto, na rede extrahospitalar, como o preconizado atualmente. As práticas assistenciais prestadas por estes serviços são as mesmas de um tradicional com o diferencial de acolhimento noturno, nos feriados e finais de semana. O CAPS III possui, no máximo, cinco leitos para eventual repouso e/ou observação. Nos casos em que o usuário encontra-se em períodos críticos, a permanência do mesmo no acolhimento noturno limita-se a sete dias corridos ou dez dias intercalados, num período de trinta dias (BRASIL, 2011).

Portanto, nota-se a importância do serviço 24 horas nos CAPSs, entendido como acolhimento noturno, na tentativa de evitar a perda da autonomia e o vínculo familiar dos usuários, gerados pela internação de longo prazo.

Segundo as Portarias SNAS n ${ }^{\circ} 224$, de 29 de janeiro de $1992^{5}$ e GM/MS n 366 , de 19 de fevereiro de 2002, ficam estabelecida a implantação do sistema 24 horas do CAPS, atendendo a proposta do cuidar em liberdade, visando tratar o cidadão no local em que vive, de forma integral, evitando abordagens que rompam com os vínculos sociais e familiares (BRASIL, 2002).

Apesar dos dados acima apresentados, estima-se que milhares de pessoas ainda vivam um processo de institucionalização permanente, sendo que este resulta na desfiliação social que é o principal resultado do projeto manicomial. Isto se deve ao fato de ser produzida socialmente uma cronicidade, além do aniquilamento de pessoas que são privadas do direito de viver em liberdade, e que são condenadas pela sua vulnerabilidade à reclusão psiquiátrica. É imperativo que, para que haja reversão deste quadro, existam equipamentos suficientes na rede intersetorial para a assistência ao portador de transtorno mental. É necessário, também, avaliar estes serviços, o processo de implementação dos mesmos e os impactos verdadeiros que trazem para o alcance dos objetivos da reforma psiquiátrica (MÂNGIA; NICÁCIO, 2003).

Assim, passamos a nos perguntar quais são as expectativas dos profissionais de saúde mental de um CAPS em relação à implantação do sistema 24 horas.

\section{OBJETIVO}

Diante o exposto, este estudo tem por objetivo analisar as expectativas dos profissionais de saúde mental de um Centro de Atenção Psicossocial quanto à implantação do serviço 24 horas.

\section{PROCEDIMENTOS METODOLÓGICOS}

Trata-se de uma pesquisa descritiva que, de acordo com Gil (1996), se caracteriza pela interrogação direta dos indivíduos sobre os significados que um determinado assunto produz em suas vidas. Esta pesquisa foi realizada em um Centro de Atenção Psicossocial, situado na cidade de São Paulo, com a participação de 11 técnicos das seguintes categorias profissionais: enfermeiras, médicos, psicólogos e assistentes sociais. Dos 24 técnicos do serviço quatro não quiseram participar da pesquisa e os outros nove profissionais estavam afastados do serviço.

Foram realizadas 11 entrevistas semi-estruturadas, gravadas após consentimento do sujeito e transcritas pelos pesquisadores.

Para a operacionalização da análise dos dados, foram utilizados os passos propostos por Minayo (1994) da seguinte forma: quanto a ordenação dos dados foi realizada a transcrição das fitas pela própria pesquisadora,

\footnotetext{
4. Portaria GM/MS nº 336, de 19 de fevereiro de 2002, que estabelece que os Centros de Atenção Psicossocial possam constituir-se nas seguintes modalidades de serviços: CAPS I, CAPS II, CAPS III definidos por ordem crescente de porte/complexidade e abrangência populacional, conforme o disposto na portaria (Ministério da Saúde, 2004).

5. Portaria SNAS n 224, de 29 de janeiro de 1992, que estabelece diretrizes e normas para os serviços de saúde do Sistema Único de Saúde e seus prestadores (Ministério da Saúde, 2004).
} 
OKAZAKI, C. et al. Acolhimento noturno: expectativas. Rev. Ter. Ocup. Univ. São Paulo, v. 21, n. 2, p. 166-173, maio/ago. 2010.

seguiu-se a leitura, e releituras do material e organizado os relatos; a classificação dos dados se deu pela caracterização da população e classificação das informações obtidas em: expectativas positivas e expectativas negativas ${ }^{6}$; na análise final realizamos uma articulação entre os dados e os referenciais teóricos da pesquisa. Para cada frase apreendida das entrevistas foi utilizado uma codificação do tipo $E$ de entrevista, 1 do primeiro sujeito entrevistado e 1, 2, 3, conforme a apreensão das frases temáticas, o que corresponde a E.1.1 (por exemplo).

Os aspectos éticos foram seguidos conforme determinado na Resolução ${ }^{\circ} 196$ de 1996, do Conselho Nacional de Saúde (CNS) (BRASIL, 1996) que trata das Diretrizes e Normas Regulamentadoras de Pesquisas em Seres Humanos, uma vez que para ser executado, o presente estudo foi submetido a aprovação na Comissão de Ética da Escola de Enfermagem da Universidade de São Paulo, Parecer n 336/2003/CEP-EEEUSP.

\section{RESULTADOS E DISCUSSÃO}

\section{1- Caracterização da população}

Dos entrevistados, 8 indivíduos eram do sexo feminino e 3 do sexo masculino, com média de idade de 38 anos, e a faixa etária prevalente entre 30-40 anos. Quanto à área de formação, foram entrevistados 2 assistentes sociais, 1 enfermeiro, 3 médicos e 5 psicólogos.

\section{2- Expectativas em relação à implantação do serviço 24 horas}

Podemos classificar as expectativas dos profissionais, quando questionados acerca da implantação do serviço, como positivas e negativas. Do total de fragmentos dos discursos identificados, que denominaremos daqui por diante de "frases", que traziam informações acerca das expectativas dos profissionais, foram obtidas 15 frases que expressavam expectativas positivas e 14 frases que expressavam expectativas negativas quanto à implantação do serviço 24 horas.

Das expectativas que consideramos como positivas, destacam-se aquelas que consideram a implantação um passo importante para o serviço, sendo mais um recurso, no arsenal terapêutico, para o tratamento das pessoas portadoras de transtorno mental grave e persistente, como podemos observar:

\footnotetext{
“...recurso a mais pra lidar com a questão da loucura...”
}

(E 2.6)

“...eu acredito muito neste dispositivo...” (E. 3.1).

“...é um dispositivo fundamental no tratamento de pacientes graves, que pretende substituir o modelo hospitalocêntrico manicomial..." (E. 3.2).

Considerando as diretrizes oficiais para a área de assistência à saúde mental no Brasil, a partir dos anos 90, do século passado, observa-se que se preconiza uma rede assistencial, como estratégia de modificação da assistência psiquiátrica centrada no hospital psiquiátrico (OLIVEIRA, 2003). Atualmente, podemos afirmar que o grande desafio está na articulação desta rede de atenção em saúde mental de base comunitária. Somente uma rede, com seu potencial de construção coletiva de soluções, é capaz de fazer face à complexidade das demandas e garantir resolutividade $\mathrm{e}$ a promoção de autonomia e cidadania das pessoas com transtornos mentais. A rede de saúde mental é complexa, diversificada, de base territorial e deve constituir-se como um conjunto vivo e concreto de referências para os usuários dos serviços, e nesta se inclui os CAPS III, de acolhimento noturno (BRASIL, 2007).

Em relação à função terapêutica dos CAPSs vários autores afirmam que os mesmos incluem projetos coletivos de grupos verbais, grupos familiares, projetos de sociabilização, intervenções individuais, atendimento psicoterápico, medicamentoso e clínico, formas de intervenções que buscam a construção e consolidação do vínculo entre os usuários e os profissionais, com objetivo de ampliar o potencial relacional daqueles e o conhecimento crítico desses, bem como diversas modalidades de recursos assistenciais e comunitários que levam a autonomia e cidadania dos usuários (PITTA et al., 1990; MÂNGIA; BARROS, 2009).

O que verificamos nas seguintes falas:

“...possibilidade de avançar na questão da reforma e na substituição mais efetiva dos hospitais psiquiátricos..." (E. 9.4).

“...dispositivo fundamental no tratamento de pacientes graves, substituir o modelo hospitalocêntrico manicomial..." (E.3.2).

“...a implantação..é fundamental para que possa efetivamente concretizar o projeto de reabilitação, da luta antimanicomial... acabando com o dispositivo manicomial

6. Foram consideradas como expectativas positivas as expressões que demonstraram vontade /interesse desse na implantação de um serviço 24 horas e, como expectativas negativas as expressões que demonstraram resistência à implantação de um serviço 24 horas. 
OKAZAKI, C. et al. Acolhimento noturno: expectativas. Rev. Ter. Ocup. Univ. São Paulo, v. 21, n. 2, p. 166-173, maio/ago. 2010.

de vez e manter as internações curtas...”(E. 3.3).

“... a principio, eu acho que é uma coisa boa, que é uma coisa que pode ajudar o serviço dar uma guinada, encontrar seu rumo, poder trabalhar cada vez mais em prol da transformação desse serviço em uma substituição viável às internações psiquiátricas..." (E. 2.3).

Quando um dos sujeitos se refere:

“... eu acho que é importante a existência de um Centro de Atenção Psicossocial 24 horas. Coincidindo com todo esse momento, com a idéia dos fechamentos de todos os manicômios.." (E.1.2).

$\mathrm{Na}$ verdade, o sujeito está se referindo à Lei $\mathrm{n}^{\circ}$ 10.216 , de 06 de abril de 2001, que dispõe sobre a proteção e os direitos das pessoas portadoras de transtornos mentais e redireciona o modelo assistencial em saúde mental (BRASIL, 2004) e a Reforma Psiquiátrica, que corresponde a um processo de trabalho que articula a formulação política de saúde mental, a inclusão social e as ações práticas desenvolvidas nos serviços (SILVA et al., 2002).

Os atores sociais, deste estudo, além de atribuírem o fato da implantação do acolhimento noturno como uma diretriz nacional, apresentam, também, contribuições para a organização da equipe profissional, o que podemos confirmar com as seguintes falas:

\footnotetext{
“..é importante como toda uma organização pensante..., com articulação de equipe..." (E.1.3)

“...desde que contratem pessoal, acho que pode ser uma alternativa interessante..." (E.5.1)
}

Os Centros de Atenção Psicossocial, segundo Pitta (1990), são dispositivos que têm como eixo central do processo de trabalho a equipe multiprofissional ${ }^{7}$, visando o atendimento da necessidade de saúde do usuário e grupos e uma relação trabalhadores / usuários mais humanizada que possibilite a cidadania.

Assim, o acolhimento noturno é representado pelos sujeitos do estudo como uma estratégia que permite, neste momento, a reorganização do serviço como o vemos.

“... tenho expectativas legais, quero o 24 horas, acho que é importante, vai ser importante, para poder dar uma continuidade, uma continência pra esse momento de maior desorganização aqui dentro, isso vai fazer toda diferença no tratamento com os pacientes..."(E.3.8).

“...que é uma coisa que pode ajudar o serviço a dar uma guinada, encontrar seu rumo...” (E.2.3)

Entendemos organização de serviço, neste estudo, além da noção de estrutura e funcionamento, também como um conjunto de ações dos trabalhadores de saúde para a produção de serviços, sobretudo, com o usuário e seu cuidado. Assim, os sujeitos demonstram:

“... possa atender o doente mental de maneira mais adequada... De uma maneira mais humana... no espaço que ele já conhece, num momento de crise em que ele não precise ir para outro lugar, aonde ele vai se deparar com uma situação mais difícil..." (E 4.1)

“...mais suporte pro paciente...” (E.7.1).

“...minha expectativa seria tanto da gente poder atender melhor os usuários...” (E.9.1).

Segundo Merhy (1999), a dimensão cuidadora visa produzir processo de falas e escutas, uma relação intercessora com o mundo subjetivo do usuário e como ele constrói nas necessidades de saúde, relação de acolhimento e vínculo, posicionamento ético e articulação de valores. Essa dimensão cuidadora esteve presente em várias falas, como podemos observar anteriormente.

Os atores também apontaram pelo fato de que a implantação representa fontes de conhecimento:

“...eu acho que é um passo importante para o Centro de Atenção Psicossocial, que vai possibilitar uma reflexão..."(E.2.1)

“... a implantação pode ser boa, do ponto de vista administrativo, e pode ser muito boa, do ponto de vista de estudo..." (E.11.2)

Em relação às expectativas negativas, a maioria dos sujeitos apresentou resistência à implantação referindo à questão salarial:

“... não acho fácil, por todas as complicações que o funcionário público passa hoje, salário...” (E.1.3).

“...profissionais, estão muito insatisfeitos com o que ganham. Para você ter um serviço 24 horas, você tem que investir mais de você..." (E.1.4).

7. Equipe multiprofissional entendida como: composição de diferentes processos de trabalho preservando as diferenças técnicas entre os trabalhadores especializados (Peduzzi, 1998) 
OKAZAKI, C. et al. Acolhimento noturno: expectativas. Rev. Ter. Ocup. Univ. São Paulo, v. 21, n. 2, p. 166-173, maio/ago. 2010.

\footnotetext{
“... não é independente de toda uma conjuntura política, $e$, sem essa conjuntura política, eu acho que a implantação do 24 horas pode significar um risco para o CAPS..." (E.2.2).
}

Segundo Tenório et al. (2000), a substituição asilar depende de um bom funcionamento de uma rede de atenção em saúde mental, rede que tem o Centro de Atenção Psicossocial como unidade estratégica, mas que inclui uma série de outros serviços que integram essa rede, como unidades psiquiátricas em hospital geral, ambulatório, unidades básicas, rede social e educacional. Como citado anteriormente, não é suficiente apenas interromper o atendimento asilar, é essencial acolher o indivíduo em um sistema que possua todos os equipamentos necessários para o atendimento de suas necessidades, incluindo, entre estas, o acolhimento noturno (BRASIL, 2011).

Entretanto os atores sociais deste estudo, além de referirem inadequação salarial, mostraram insegurança quanto ao suporte da rede de apoio, pois alegam que o serviço público de saúde passa por dificuldades. Fica claro também que os sujeitos não vêem um efetivo grau de integração entre o serviço e a rede.

“...trabalho que é fundamental, mas sem apoio de uma rede, sem uma rede efetiva..." (E.3.7).

“...preocupação... porque eu acho que o serviço público, no Brasil, está muito devagar e muito carente de serviços, de pessoas..." (E.4.2)

A Organização Mundial da Saúde (2001) diz que a política deve fixar prioridades e delinear abordagens, com base nas necessidades identificadas e levando em conta os recursos disponíveis. Nos últimos anos a expansão e a consolidação e qualificação da rede de atenção à saúde mental, sobretudo dos Centros de Atenção Psicossocial (CAPS), foram objetivos principais do Ministério da Saúde. Os CAPSs são estratégicos para a organização da rede de atenção à saúde mental num determinado território e a expansão destes serviços é fundamental para mudar o cenário da atenção à saúde mental no Brasil (BRASIL, 2007), sobretudo, na cidade de São Paulo e no Estado de São Paulo. Assim, o grande desafio é a articulação desta rede.

“... que tem muitas deficiências, pensando que o Estado investe nesses serviços..." (E.8.1).

Ressaltamos o Estado de São Paulo por representar o cenário deste estudo e ainda apresentar o maior número de leitos psiquiátricos do país, sendo o Estado que detém a maior proporção de moradores para o total de leitos (Brasil, 2011), são 6400 moradores, cerca de $60 \%$ do total dos leitos do Estado (BARROS; BICHAF, 2008). A iniciativa da Secretaria de Estado de São Paulo, em realizar o "Censo Psicossocial dos Moradores de Hospitais Psiquiátricos do
Estado de São Paulo" foi um fato relevante por apontar onde se concentram os moradores e permitir pactuações locais para o processo de desinstitucionalização destes pacientes, com expansão de CAPS, sobretudo CAPS III e implantação de outros recursos para tal processo, o que pode significar avanços da rede substitutiva, entretanto, após dois anos do lançamento de seus resultados, menos de 300 moradores foram desinstitucionalizados, o que nos apontam para os obstáculos políticos e técnicos que o processo de reinserção social de moradores ainda apresentam para a Reforma Psiquiátrica brasileira (BRASIL, 2011).

Finalmente, alguns atores sociais referem que o serviço substitutivo não deve transformar-se em estrutura manicomial tais como:

“... na prática tem que ir construindo, para não virar uma emergência psiquiátrica...” (E.6.1).

“... o risco é de transformar numa instituição que possa bancar tudo, como uma instituição total, como um manicômio, isso é um risco..." (E.2.5).

Para que os serviços possam rever sobre suas práticas e seu projeto institucional, o Ministério da Saúde instituiu a partir de 2004 o Programa Nacional de Avaliação dos Centros de Atenção Psicossocial - Avaliar CAPS, que tem por objetivos consolidar as informações sobre os CAPSs; caracterizar os serviços; acompanhar a rede; aferir a qualidade da assistência prestada e desenvolver indicadores (BRASIL, 2011).

Os resultados da avaliação de 2008/2009 mostraram que a maioria dos serviços caminha na direção de uma clínica de atenção psicossocial aberta para o território, na busca de acompanhar o usuário em suas diversas situações de vida e com a expectativa de cumprir as diferentes missões dos CAPSs, entretanto, apontam que em termos de estrutura dos serviços precisa melhorar muito, sobretudo no que diz respeito aos recursos humanos (BRASIL, 2011).

Assim, para que os trabalhadores dos serviços possam refletir sobre suas práticas e o projeto institucional, a avaliação deve ser constante e, sempre estar pautada num processo de trabalho baseado em projetos, metas, prazos e recursos bem definidos, reunindo saberes e pessoas de diversas áreas e especialidades diferentes.

\section{CONSIDERAÇÕES FINAIS}

Por meio deste estudo, pudemos verificar que é de extrema importância a implantação do acolhimento noturno, levando em consideração todo contexto de Reforma Psiquiátrica que o sistema de saúde vem passando desde o último século. O movimento de Reforma Psiquiátrica 
OKAZAKI, C. et al. Acolhimento noturno: expectativas. Rev. Ter. Ocup. Univ. São Paulo, v. 21, n. 2, p. 166-173, maio/ago. 2010.

inseriu o tema da desinstitucionalização na pauta da política pública e, consequentemente, o da mudança radical no modelo de atenção à saúde mental. Essa mudança se liga às estratégias de difusão e expansão das inovações institucionais desenvolvidas no campo da saúde mental, como os Centros de Atenção Psicossociais.

$\mathrm{Na}$ análise das expectativas positivas, verificou-se que a implantação do serviço de acolhimento noturno é um passo importante para a instituição, pois será mais um arsenal terapêutico no tratamento das pessoas portadoras de transtorno mental grave e persistente. Outro aspecto positivo encontrado foi que os atores sociais relataram que haveria maior articulação das equipes no caso de implantação e, consequentemente, maior organização da estrutura e funcionamento do serviço, impedindo a desvinculação do usuário desta estrutura, podendo assim, ter uma maior relação com a rede, que é composta por diversos equipamentos que devem se combinar ou se completar e, sobretudo se articular.

Analisando as expectativas negativas, os atores sociais demonstraram a insatisfação quanto à questão salarial e à insegurança sobre o suporte da rede de apoio, pelas dificuldades que o serviço público de saúde está passa.

Em ambas as expectativas, o tema de maior enfoque foi a rede de saúde mental que se encontra fragmentada e desarticulada. Portanto, ressaltamos a necessidade de um aperfeiçoamento da articulação de rede, tanto no âmbito local (equipe) quanto no sistema (apoio). É necessário, para isso, um repensar das equipes quanto à estrutura institucional, em que a mesma possa buscar novas formas de compreensão e intervenção na viabilização de uma comunicação efetiva e articulada entre equipe e a rede de atenção em saúde mental.

O acolhimento noturno não apresenta somente um diferencial no formato institucional, mas também faz diferença no que se refere à reflexão de uma concepção teórica, que engloba a desinstitucionalização e a clínica ampliada bem como na forma de organização do cotidiano institucional, ou seja, a reorganização do processo de trabalho dos profissionais envolvidos.

OKAZAKI, C.; OLIVEIRA, M. A. F.; CLARO, H. G.; PAGLIONE, H. B.; SOARES, R. User embracement: expectations of the mental health professionals. Rev. Ter. Ocup. Univ. São Paulo, v. 21, n. 2, p. 166-173, maio/ago. 2010.

\begin{abstract}
This study had as objective to identify the expectations of the mental health professional from the Psychosocial Attention Center, the city of São Paulo, as regards the implantation of a 24-hours service (user embracement). This is about a descriptive research that is characterized by the direct interrogation of the individuals about the meaning that a determined subject produces in their lives. Eleven actors participated in this study, from the categories: nurses, doctors, psychologists and social assistants. For the data it were followed the hermeneutic dialectics steps: sorting, grading and final analysis. It was realized the characterization of the population and the classification of the information obtained in positive and negative expectations. The final analysis considers that the implantation of a user embracement: service represents one more resource in the therapeutic arsenal for the treatment of people who have severe and persistent mental bewilderment that allows the continuous attention.
\end{abstract}

KEY WORDS: User embracement; Psychiatry; Mental health; Health services/manpower; Mental health services/manpower; Health personnel.

\title{
REFERÊNCIAS
}

ARANHA E SILVA, A. L. O Projeto Copiadora do CAPS: do trabalho de reproduzir coisas à produção de vida. 1997. Dissertação - Escola de Enfermagem, Universidade de São Paulo, São Paulo, 1997.

BRASIL. Ministério da Saúde. Legislação em saúde-mental 1990-2002. $3^{\text {a }}$ ed. rev. Atual. Brasília DF, 2002.

BRASIL. Ministério da Saúde. Legislação em saúde mental

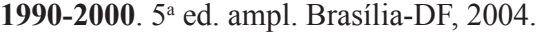

BRASIL. Ministério da Saúde. Relatório de Gestão 2003-2006 - Saúde Mental no SUS: acesso ao tratamento e mudança do modelo de atenção. Relatório de Gestão 2003-2006. Brasília - DF: Ministério da Saúde, 2007.

BRASIL. Ministério da Saúde. Relatório de Gestão 2007-2010 saúde mental no SUS: as novas fronteiras da reforma psiquiátrica. 
Brasília (DF), 2011.

GIL, A. C. Como elaborar projetos de pesquisa. 3a ed. São Paulo : Atlas, 1991.

MÂNGIA, E. F; BARROS, J. O. Projetos terapêuticos e serviços de saúde mental: caminhos para a construção de novas tecnologias de cuidado. Rev. Ter. Ocup. Univ. São Paulo, v. 20, n. 2, 2009.

MÂNGIA, E. F.; NICÁCIO, F. De volta para Casa: reconhecendo o direito de viver fora dos manicômios. Rev. Ter. Ocup. Univ. São Paulo, v. 14, n. 3, 2003.

MINAYO, M. C. S. Pesquisa social: teoria, método e criatividade. Petrópolis: Vozes, 1994.

MERHY, E. E.; FRANCO, T. B.; BUENO, W. S. O acolhimento e os processos de trabalho em saúde: o caso Betim (MG). Cad Saúde Pública, v. 15, n. 2, p. 345-53, 1999.

OLIVEIRA, A. G. B.; ALESSI, N. P. A reforma psiquiátrica e o processo de trabalho das equipes de saúde mental. Rerv Paul. Enf., v. 22, n. 1, p. 31-42, 2003.

ORGANIZAÇÃO MUNDIAL DA SAÚDE. Organização Panamericana da Saúde. Relatório sobre a saúde no mundo 2001Saúde Mental: nova concepção, nova esperança. Genebra, 2001.

PEDUZZI, M. Equipe multiprofissional de saúde: a interface entre trabalho e interação. Dissertação (Doutorado Medicina)Departamento de Medicina Preventiva e Social, Faculdade Ciências Médicas, Universidade Estadual de Campinas. Campinas, 1998.

PITTA, A. M. F.; COSTA, A. L.; CORAZZA, D. M.; LIMA, E.; GOLDBERG, J. I.; FERNANDES, M. H.; REPACH, N. A.; YASSUI, S. Avaliação de serviços de atenção médicopsicossocial a usuários do sistema de saúde no município de São Paulo. Relatório de pesquisa. São Paulo, NEP-CAPS/DMPFMUSP/Instituto de Saúde, SES/SP/Banco Mundial, 1990.

PITTA, A. M. F. Os centros de atenção psicossocial: espaço de reabilitação. J Bras. Psiquiatr., v. 43, n. 12, p. 647-654, 1994.

SARACENO, B. Libertando identidades: da reabilitação psicossocial à cidadania possível. Belo Horizonte. Ed. Te Corá. 1999.

SILVA, A. T. M. C.; BARROS, S.; OLIVEIRA, M. A. F. Políticas de saúde e saúde mental no Brasil: a exclusão/inclusão social como intervenção e gesto. Rev. Esc. Enf. Univ. São Paulo, v.36, n. 1, p.4-9, 2002.

TENÓRIO, F.; OLIVEIRA, R.; LEVCOVITZ, S. A importância dos dispositivos de recepção. Cad. IPUB, Rio de Janeiro, v. 4, n. 7, p. 7-14. 2000. 